\title{
Conformance testing trials on ERMES receivers
}

\author{
P. Kostarakis, A. A. Alexandridis, K. Dangakis, A. Vlahakis, \\ $P$. Katrivanos, F. Lazarakis, A. Paschalis and N. Gaitanis \\ Inst. of Informatics and Telecommunications, NCSR "Demokritos", \\ 15310 Aghia Paraskevi, Athens, GREECE, \\ Tel. No.: + 3016520847 , Fax No.: + 3016532175 , \\ E-mail:aalex@iit.nrcps.ariadne-t.gr
}

\begin{abstract}
A test system for checking the receivers of the digital pan-European paging system ERMES for conformity to the standard specification for air interface and receiver operation has been developed according to the ISO 9646 standardized methodology for conformance testing services. The test procedures, various test setups and test execution software, implemented and integrated in a fully operational test system, are described in this paper. Complete test suites have been carried out on ERMES receivers. A part of the results of test trials is listed here, demonstrating the test system functionality.
\end{abstract}

Paging system, ERMES, conformance testing

\section{Keywords}

\section{INTRODUCTION}

Conformance testing is the verification that an implementation meets the formal requirements of the referenced standard. Its primary objective is to ensure interoperability among different product implementations. The need for a variety of interconnection and interoperability schemes among different implementations has led to the establishment of a standardized methodology and modeling approach for the description of the operation of the introduced systems and services, namely the Open System Interconnection (OSI) model. 
The operational capabilities of any system or commercial product, derived through the realization of a new concept, are fully described in the relevant standard specification which may be considered as national, international, licensed, etc. Such implementations, which usually come from various manufacturers and, sometimes, even technologies, can be tested uniformly to determine whether they conform to these standards. Consequently, authorized organizations should ensure the existence of accredited laboratories to provide Conformance Testing Services (CTS) for the industry.

In this paper, we describe the development of a Conformance Test System" for the air interface and receiver operation of the European paging system ERMES (European Radio MEssage System), as specified by the European Telecommunications Standards Institute (ETSI) (prETS 300 133-1, prETS 300 133-4, prETS 300 133-5, 1991). In particular the test procedures, with the various test setups and the test execution software, are described. Finally, a detailed list of results, obtained from trials covering key test cases, is included.

\section{ERMES OVERVIEW}

ERMES is allocated on a common frequency band throughout Europe comprising sixteen 25 $\mathrm{kHz}$ spaced channels between $169.425 \mathrm{MHz}$ and $169.800 \mathrm{MHz}$. The ERMES network operates at a data rate equal to $6.25 \mathrm{kbits} / \mathrm{s}$, which is reduced at a symbol rate equal to 3.125 kbaud by the use of 4-PAM/FM modulation. Normally, an ERMES receiver will receive calls on a single channel used by its home network. However, it will scan all the 16 channels in the case when that single channel is lost (e.g., during roaming).

The structure of the ERMES transmission protocol is based on a sequence of 60 minutes total duration comprising 60 paging cycles. Sequences are coordinated with the UTC (Universal Time Coordinated) so that on the hour a new sequence commences. Each cycle (one minute long) is divided into five subsequences commencing at 12 second intervals. Each subsequence is further divided into 16 batches and each batch has four partitions (synchronization, system information, address and message partitions). The first fifteen batches in every subsequence have a length of 154 codewords and the final batch has a length of 190 codewords (standard information unit of 30 bits length). The receiver population is divided into 16 groups and each receiver is allocated to one of the 16 batch types according to the 4 least significant bits of its basic Radio Identity Code (RIC).

The ERMES system supports the following four paging categories:

- Tone only: the receiver shall respond to at least one of eight alert types.

- Numeric: the receiver shall provide for the reception of a 20 digit numeric message.

- Alphanumeric: the receiver shall provide for the reception of a 400 character text message.

- Transparent data: the receiver shall provide for the reception of an arbitrary data message. In addition, a wide range of supplementary services is also supported, e.g., acknowledgement, protection against loss of messages, three levels of priority, long messages.

"This work has been co-funded by the European Union and carried out under the CTS-3/ ERMES project, contract No. $90 / 45067$. 
TEST PROCEDURES
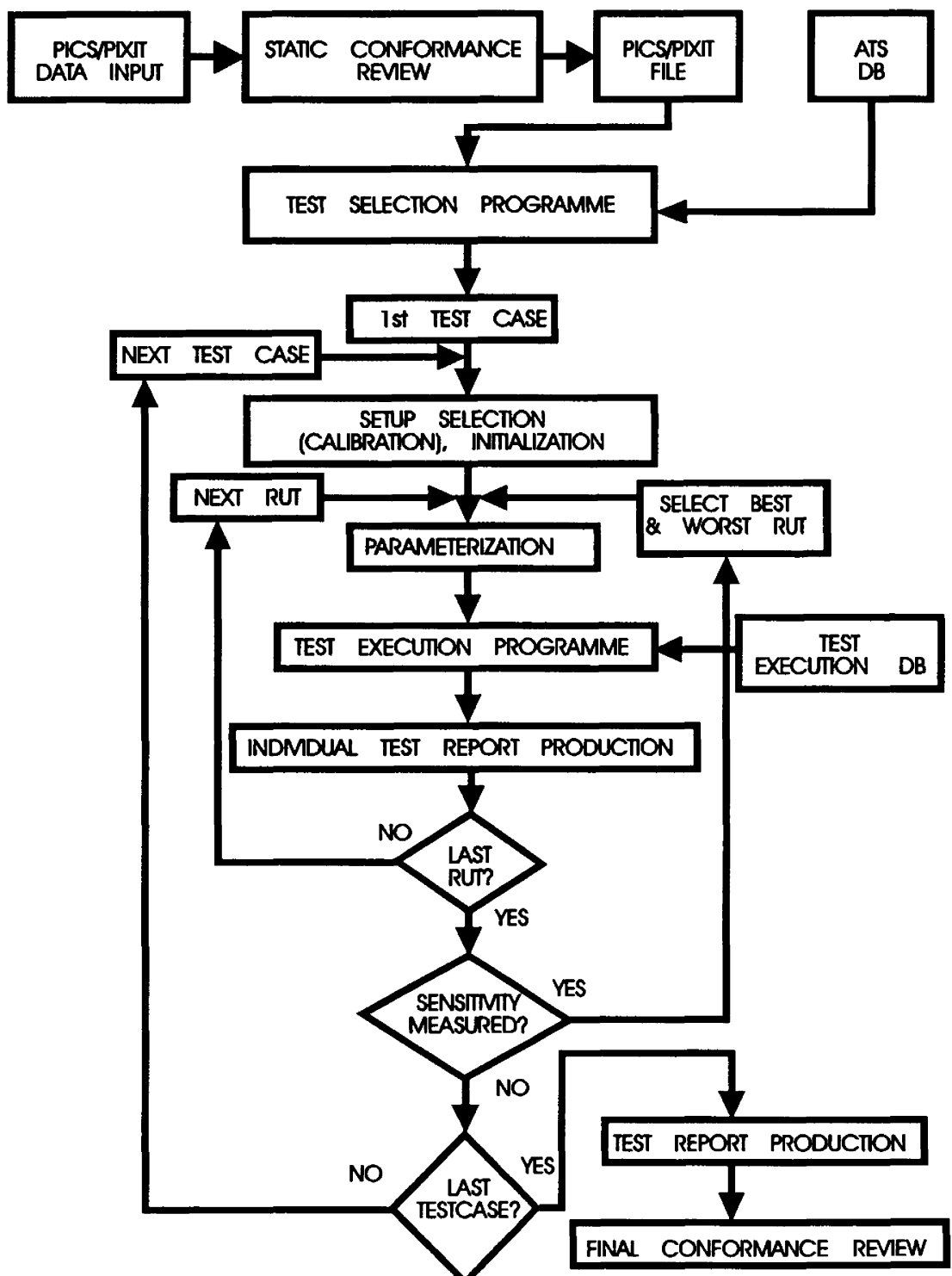

Figure 1 Complete Test Procedure Flow Chart. 


\section{TEST PROCEDURES}

The procedures carried out by the test system in order to nu the tests are shown in the flow chart of Figure 1. A more detailed description is given in (CTS-3 ERMES Project 45067, Deliverable 3, 1992) and (Dangakis, 1992). PICS (Protocol Implementation Conformance Statement) and PIXIT (Protocol Implementation eXtra Information for Testing) contain the required information for test selection and parameterisation (CTS-3 ERMES Project 45067, Deliverable 5, 1992). The data included in the PICS and PIXIT proformas will be stored in a file in order to allow an automated selection and parameterisation procedure to be performed. The programme that enables the data input performs also some consistency checks ("static conformance review"). The whole ERMES Abstract Test Suite (ATS) is organized in an ERMES database (CTS-3 ERMES Project 45067, Deliverable 1, 1991). The "test selection programme", based on the PICS and PIXIT information, handles the ATS database and produces the selected ATS containing all the test cases relevant to the particular Receiver Under Test (RUT). For each selected test case, the file produced by the "test selection programme" contains all the necessary information for the test execution. First the setup, that is appropriate for the particular test case under consideration, is selected and the necessary installation and preparations are done (ETSI PT8, Final Report, 1990).

Several parameters must be specified before running a test. Some of them are derived from the RIC that is specified, for each one of the six receivers to be tested, by the ERMES standard (prETS 300 133-5, 1991). Most of the test cases require that only the best and the worst (with respect to the measured average usable sensitivity) receivers of the six supplied shall be used. This means that the sensitivity measurement tests shall be carried out first, and then their results shall be taken into account during the test case selection and parameterisation. Some parameters are specified according to the input information from PICS / PIXIT. Others are set according to the requirements of the particular test case and their values are retrieved by the "test execution programme" from the "test execution database". Finally, there are some parameters that need to be set and updated in real time, while running the tests.

The different setups that are used and the "test execution programme" (software tool actually running the tests) are described in the next two sections.

\section{SETUPS OF THE TEST SYSTEM}

According to the standards (prETS 300 133-5, 1991), some tests are carried out on an Open Area Test Site (OATS) while the rest are carried out using a test fixture (TEM-cell). For extreme test conditions a test fixture placed in a climate chamber is also required. So, the following four setups were used in the test system:

- setup A for OATS measurements;

- setups B, C and D for measurements using a TEM-cell.

The block diagrams of these setups are given in Figure 2, where a RF signal generator simulates the ERMES base station. The calibration of the test site and test fixture (ETSI PT8, Final Report, 1990) establishes a relationship between the output level of the RF signal 
generator and the field strength applied to the RUT. This relationship for each one of the setups is given below.

\section{Test setup A}

The following formula was applied, giving the field strength $e$ at the RUT position:

$e=u+\mathrm{AF}+\mathrm{AL}+\mathrm{CL}(\mathrm{dB} \mu \mathrm{V} / \mathrm{m})$

where

$u \quad$ : substitution antenna output measured with spectrum analyzer $(\mathrm{dB} \mu \mathrm{V})$

$\mathrm{AF}=12.9 \mathrm{~dB}:$ substitution antenna factor

$\mathrm{AL}=0.2 \mathrm{~dB} \quad$ : substitution antenna loss

$\mathrm{CL}=0.9 \mathrm{~dB} \quad$ : cable loss, from substitution antenna to spectrum analyzer

\section{Test setup B}

According to the calibration of the TEM cell, the average usable sensitivity $(\mathrm{dB} \mu \mathrm{V} / \mathrm{m})$ equals to the average of recorded values minus $9 \mathrm{~dB}$.

\section{Test setups $C \& D$}

RF generator A (wanted signal) output level, $r$, adjustment:

The results from the measurements of the average usable sensitivity, in the OATS and the test fixture, are used to calculate the $r(\mathrm{~dB} \mu \mathrm{V})$ corresponding to a field strength of $25 \mathrm{~dB} \mu \mathrm{V} / \mathrm{m}$, in the test fixture. The following formula was applied:

$r=l+d+\mathrm{A}$

where

l: RF Generator A output level $(\mathrm{dB} \mu \mathrm{V})$ obtained from the measurement of the average usable sensitivity in the test fixture.

$d$ : Difference (in $\mathrm{dB}$ ) between the measured average usable sensitivity in OATS and the reference figure $(25 \mathrm{~dB} \mu \mathrm{V} / \mathrm{m})$.

A: Additional attenuation (in $\mathrm{dB}$ ) due to combiner and extra cables (equal to $7.7 \mathrm{~dB}$ for setup C and to $10 \mathrm{~dB}$ for setup $\mathrm{D}$ ).

\section{TEST EXECUTION PROGRAMME}

This software runs on the main controller (PC) and communicates with the other devices through digital $\mathrm{I} / \mathrm{O}$, serial and IEEE interface cards. Before running a specific test case, it initializes the setup by:

- Reading the ambient temperature and relative humidity values and verifying that they are within the limits specified by the standard for normal test conditions.

- Setting the climate chamber to the extreme temperature and relative humidity values specified by the standard for extreme test conditions (if needed). 

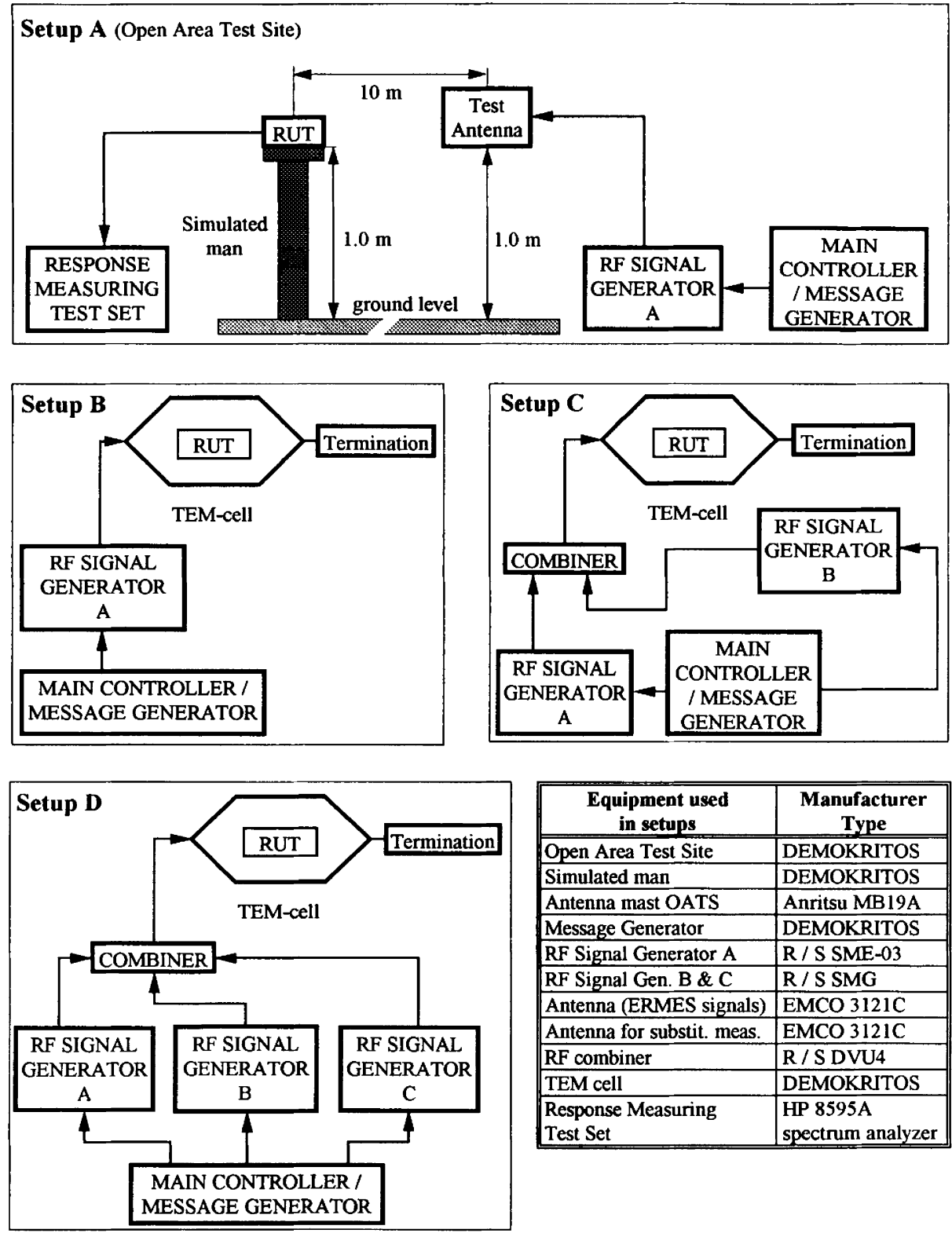

\begin{tabular}{|l|l|}
\hline \multicolumn{1}{|c|}{$\begin{array}{c}\text { Equipment used } \\
\text { in setups }\end{array}$} & \multicolumn{1}{c|}{$\begin{array}{c}\text { Manufacturer } \\
\text { Type }\end{array}$} \\
\hline \hline Open Area Test Site & DEMOKRITOS \\
\hline Simulated man & DEMOKRITOS \\
\hline Antenna mast OATS & Anritsu MB19A \\
\hline Message Generator & DEMOKRITOS \\
\hline RF Signal Generator A & R / S SME-03 \\
\hline RF Signal Gen. B \& C & R / S SMG \\
\hline Antenna (ERMES signals) & EMCO 3121C \\
\hline Antenna for substit. meas. & EMCO 3121C \\
\hline RF combiner & R / S DVU4 \\
\hline TEM cell & DEMOKRITOS \\
\hline $\begin{array}{l}\text { Response Measuring } \\
\text { Test Set }\end{array}$ & $\begin{array}{l}\text { HP 8595A } \\
\text { spectrum analyzer }\end{array}$ \\
\hline
\end{tabular}

Figure 2 Block diagram of Test System Setups and list of equipment. 
- Setting the power supply voltage to the nominal or extreme (maximum or minimum) value.

- Setting the center frequency of the RF signal generator to the specified RF channel.

- Setting the RF signal generator output power level to the proper value.

During most of the considered test cases, the common and basic task next carried out by the test execution software is the transmission of a burst containing a message at a specified time slot (batch). In order to do this, the software must step through the following:

- It forms the information content that is to be transmitted, by combining information retrieved from PICS / PIXIT and the test execution database or entered by operator.

- Time related information (date, hour, cycle, subsequence and batch numbers) is also inserted and updated in real time.

- Error correction coding, where the information bits are grouped in codewords, is done followed by codeword interleaving.

- The resulting bit-stream buffered in the PC RAM, is then passed, at the nominal bit-rate and through the digital $\mathrm{V} / \mathrm{O}$ card, to the modulation data input of the RF signal generator performing ERMES modulation. The software uses for synchronization a time reference obtained from a UTC source (Odetics GPStar).

The above steps, resulting in the transmission of an ERMES message (single batch transmission) at a specified RF channel frequency, may be repeated several times while running a single test case, depending on the requirements. This procedure is automatically repeated but, after each transmission, the software waits for the operator's input denoting if the response was successful or not.

The ERMES conformance testing services may be divided in two categories: the RF parameter tests and the protocol conformance tests (CTS-3 ERMES Project 45067, Deliverable 5, 1992).

The first category includes all test cases directly related to Radio Frequency operational characteristics of ERMES receivers. The value of the RF parameter under test is derived statistically from the RF signal levels recorded during repeated single-batch transmissions. This value is then compared with the corresponding verdict criterion, in order to assign the verdict (Pass or Fail) to the specific test.

To illustrate such a procedure, we consider, as an example, the test case concerning the measurement of average usable sensitivity that corresponds to a "message acceptance ratio of 80\%", as defined in Annex A, subclause A.1.2. of (prETS 300 133-5, 1991). The software uses the algorithm shown in the flow chart of Figure 3. This procedure is repeated for the 8 positions, $45^{\circ}$ apart, of the receiver. The corresponding average values of the generator output are determined and noted. Using the calibration of the test site, the 8 field strengths corresponding to the 8 average values are calculated and the average measured usable sensitivity is derived as a mean field strength.

The second category (protocol conformance tests) includes all test cases referred to the essential or optional features of ERMES receiver operation, with respect to its particular paging type. The test execution software manages more complex message transmissions covering multiple batches, subsequences (16 batches) and RF channels. In this category, generally, one message transmission occurs and the verdict is derived by comparing the receiver response to the expected one. 


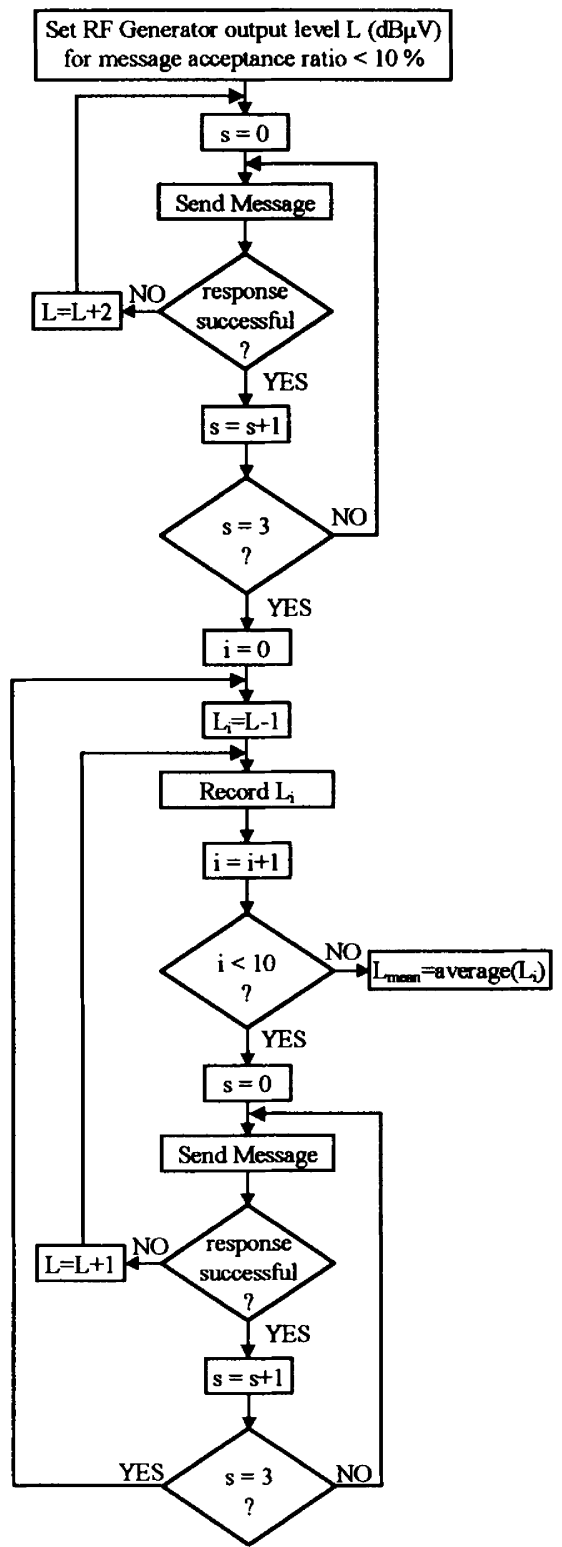

Figure 3 Flow chart of the algorithm for the implementation of a message acceptance ratio of approximately $80 \%$. 
Table 1 List of covered test cases with the corresponding ATS No. and setup

\begin{tabular}{|c|c|c|}
\hline$A T S N o$. & SETUP & DESCRIPTION \\
\hline $001-003$ & A & Average Usable Sensitivity channel 8 \\
\hline 004-006 & B & Average Usable Sensitivity channels $0,8,15$ \\
\hline $007-009$ & B & Average Usable Sensitivity with tx freq. offset \\
\hline 010-012 & B & Average Usable Sensitivity (extr. conditions) \\
\hline $013-015$ & B & Average Us. Sens. (extr. cond.+freq. offset) \\
\hline 016-018 & B & Average Usable Sensitivity channel switching \\
\hline $020-022$ & A & Usable Input Level Range \\
\hline $023-025$ & $\mathrm{C}$ & Co-channel Rejection \\
\hline $026-028$ & $\mathrm{C}$ & Adjacent channel Selectivity (norm. cond.) \\
\hline 029-031 & $\mathrm{C}$ & Adjacent channel Selectivity (extr. cond.) \\
\hline $032-034$ & $\mathrm{C}$ & Spurious Response Immunity \\
\hline $035-037$ & D & Intermodulation Immunity \\
\hline $038-040$ & $\mathrm{C}$ & Blocking Immunity \\
\hline $044-046$ & B & First Message in Batch \\
\hline 047-049 & B & Last Message in Batch \\
\hline $050-052$ & B & Message in Next Batch \\
\hline $053-055$ & B & Message in Last Available Batch \\
\hline $056-058$ & $\mathbf{B}$ & Tenure of Message \\
\hline $059-061$ & B & Two Messages in the Same Batch \\
\hline $062-064$ & B & Message in the next Subsequence \\
\hline $065-067$ & B & Message reception on all ERMES channels \\
\hline $068-070$ & B & Recognition of Zone Code \\
\hline $071-073$ & B & Two Messages in the Same Batch \\
\hline $080-081$ & B & Maximum length message \\
\hline $082-084$ & B & Alert (Normal operation) \\
\hline $085-087$ & B & Alert (Silent mode) \\
\hline 088 & B & Termination of numeric messages \\
\hline 089 & B & Termination of alphanumeric messages \\
\hline $090-095$ & B & Repeated Call Indication \\
\hline 096-098 & B & Group Call \\
\hline $099-101$ & B & Tenure of Group Message \\
\hline $102-103$ & B & Low Battery Indication \\
\hline $111-113$ & B & RSVD bits \\
\hline $116-119$ & B & Indication of lost message \\
\hline $120-122$ & B & Urgent message indicator \\
\hline
\end{tabular}


Table 2 List of results for test cases concerning RF parameters measurements (alphanumeric paging category).

\begin{tabular}{lllcc}
\hline ATS & Measured Value & Verdict Criteria & \multicolumn{2}{c}{ Verdict } \\
\cline { 3 - 4 } No. & & & $\checkmark$ & PAIL \\
\hline 022 & 4 correct messages out of 4 & 4 correct messages out of 4 & $\checkmark$ \\
025 & $7.6 \mathrm{~dB}$ & $\leq 10 \mathrm{~dB}$ & $\checkmark / \checkmark$ \\
034 & $79.2 / 83.2 \mathrm{~dB} \mu \mathrm{V} / \mathrm{m}$ & $\geq 76 \mathrm{~dB} \mu \mathrm{V} / \mathrm{m}$ & $\checkmark / \checkmark$ \\
037 & $73.9 / 73.7 \mathrm{~dB} \mu \mathrm{V} / \mathrm{m}$ & $\geq 70 \mathrm{~dB} \mu \mathrm{V} / \mathrm{m}$ & $\checkmark / \checkmark$ \\
040 & $103.4 / 109.0 \mathrm{~dB} \mu \mathrm{V} / \mathrm{m}$ & $\geq 84 \mathrm{~dB} \mu \mathrm{V} / \mathrm{m}$ & $\checkmark$ & \\
003 & $21.8 \mathrm{~dB} \mu \mathrm{V} / \mathrm{m}$ & $\leq 25 \mathrm{~dB} \mu \mathrm{V} / \mathrm{m}$ & $\checkmark / \checkmark$ \\
006 & $22.7 / 24.3 \mathrm{~dB} \mu \mathrm{V} / \mathrm{m}$ & $\leq 25 \mathrm{~dB} \mu \mathrm{V} / \mathrm{m}$ & $\checkmark / \checkmark$ & \\
009 & $24.1 / 20.5 \mathrm{~dB} \mu \mathrm{V} / \mathrm{m}$ & $\leq 25 \mathrm{~dB} \mu \mathrm{V} / \mathrm{m}$ & & $\checkmark$ \\
012 & No RUT response & $\leq 31 \mathrm{~dB} \mu \mathrm{V} / \mathrm{m}$ & & $\checkmark$ \\
015 & No RUT response & $\leq 31 \mathrm{~dB} \mu \mathrm{V} / \mathrm{m}$ & & $\checkmark$ \\
018 & No RUT response & $\leq 25 \mathrm{~dB} \mu \mathrm{V} / \mathrm{m}$ & & $\checkmark$ \\
028 & $54.8 \mathrm{~dB}$ & $\geq 60 \mathrm{~dB}$ & & \\
031 & No RUT response & $\geq 50 \mathrm{~dB}$ & & \\
\hline
\end{tabular}

Table 3 List of results for test cases concerning Protocol Conformance tests (alphanumeric paging category).

\begin{tabular}{llc}
\hline ATS & Verdict Criteria & Verdict \\
No. & PASS FAIL \\
\hline 046 Correct reception without error & $\checkmark$ \\
049 & Correct reception without error & $\checkmark$ \\
052 & Correct reception without error & $\checkmark$ \\
055 & Correct reception without error & $\checkmark$ \\
058 & No reception & $\checkmark$ \\
061 & Correct reception of both messages without error & $\checkmark$ \\
064 & Correct reception without error & $\checkmark$ \\
067 & Correct reception on all ERMES channels without error & $\checkmark$ \\
070 & No reception & $\checkmark$ \\
073 & Correct reception of message 2 without error & $\checkmark$ \\
081 & Correct reception without error & $\checkmark$ \\
084 & Correct reception without error giving the appropriate alert & $\checkmark$ \\
087 & Correct reception without error (no audible alert) & $\checkmark$ \\
088 & Presentation of only the first 4 characters & $\checkmark$ \\
089 & Correct reception without error & \\
095 & Correct reception without error, giving a repeated call indication & \\
098 & Correct reception without error & $\checkmark$ \\
101 & No reception of group message & $\checkmark$ \\
103 & $\geq 1.08$ V & $\checkmark$ \\
113 & Correct reception without error & $\checkmark$ \\
119 & The receiver shall indicate that messages are missing & $\checkmark$ \\
122 & Correct reception without error, with an urgent message indication & $\checkmark$ \\
\hline
\end{tabular}


When the execution of a test case has finished and a verdict (Pass, Fail) is assigned to it, the test results are stored. This information is updated at the end of execution of each test case and is used to produce the test report at the end of the test campaign (Figure 1).

The verification and the calibration of the ERMES test system is performed using the ERMES Reference Implementation (RI), (CTS-3 ERMES Project 45067, Deliverable 7, 1993). The ERMES RI is a device that consists a reference system implemented in accordance to the ERMES standard. It proves the equivalence of the testing tools as well as the functionality of the CTS centers, prior to approvals and commercial testing.

The ERMES test system presented here is currently operational in our laboratory. Test suites covering the test cases listed in Table 1 have been carried out on ERMES receivers coming from various manufacturers. In order to demonstrate the operation of the implemented ERMES test system, we present (in Tables 2,3 ) the results of some test cases applied on a specific prototype ERMES receiver for alphanumeric paging category. The information that this receiver failed in some tests, could be useful in modification of the design of the particular product, in order to meet the ERMES standard requirements completely.

\section{CONCLUSION}

In this paper, the development of a Conformance Test System for the ERMES air interface and receiver operation was presented and the procedures for running the tests were described. Complete test suites have been carried out on ERMES receivers coming from various manufacturers and a list of indicative results was given, demonstrating the operation of the system in accordance to the ISO 9646 standardized methodology for conformance testing services (ISO/IEC 9646, 1993) and (EWOS / ETSI PT5, 1990).

\section{REFERENCES}

CTS-3 ERMES Project 45067, Deliverable 1, Version 2, (1991) "ERMES Test Specification, Abstract Test Suite".

CTS-3 ERMES Project 45067, Deliverable 3, Final Version, (1992), "ERMES Test Specification, Test Methodology and Procedures".

CTS-3 ERMES Project 45067, Deliverable 5, Final Version, (1992), "Test Executive Software".

CTS-3 ERMES Project 45067, Deliverable 7, Final Version, (1993), "ERMES Reference Implementation".

Dangakis, K., Tombras, G., Paschalis, A., Papavramidis, A. and Kostarakis, P., (1992) "Development of a Conformance Test System for ERMES Receivers", Proceedings of The Third IEEE International Symposium on Personal, Indoor and Mobile Radio Communications, Boston, Massachusetts.

ETSI PT8, Final Report, (1990), "Methods of Measurement for Mobile Radio Equipment". 
EWOS / ETSI PT5, (1990), "Technical Report on OSI Conformance Testing Methodology and Procedures in Europe".

ISO/IEC 9646, (1993), "IT - OSI - Conformance Testing Methodology and Framework".

prETS 300 133-1, (1991), "Paging Systems; European Radio Message System (ERMES), Part 1 - General Aspects".

prETS 300 133-4, (1991), "Paging Systems; European Radio Message System (ERMES), Part 4 - Air Interface Specification".

prETS 300 133-5, (1991), "Paging Systems; European Radio Message System (ERMES), Part 5 - Receiver Conformance Specification". 